\title{
NUEVA NORMATIVA DE DIVULGACIÓN DE INFORMACIÓN NO FINANCIERA PARA GRANDES EMPRESAS. ANÁLISIS DE CASOS SOBRE POLÍTICAS DE CONCILIACIÓN DE LA VIDA LABORAL, PERSONAL Y FAMILIAR
}

\author{
New regulations on the disclosure of non-financial information for large \\ companies. Analysis of cases on work-life balance policies
}

Kamilla Teixeira-Silva, Ainhoa Saitua-Iribar*

Universidad del País Vasco / Euskal Herriko Unibertsitatea, España

\section{RESUMEN}

La directiva 2014/95/UE, aplicable a las empresas más grandes, exige la presentación de un Estado de Información No financiera que explique las políticas relacionadas con aspectos sociales y las medidas adoptadas incluyendo, entre otros, las relativas a la conciliación familiar como parte de las condiciones de trabajo. En este estudio cualitativo exploratorio de casos, se analiza la información divulgada por cuatro empresas que pueden considerarse especialmente sensibles al tema de la conciliación familiar en el primer ejercicio de aplicación del RDL 18/2017 en España, y se lleva a cabo una selección de buenas prácticas de divulgación que puedan servir de guía para otras empresas que quieran aplicarlo, incluso sin estar obligadas. Los resultados confirman el efecto positivo de la nueva normativa en el nivel de información divulgada respecto al ejercicio anterior, pero sugieren que aún hay margen de mejora en la cantidad y calidad de la información sobre conciliación divulgada por las empresas.

Palabras clave: Responsabilidad Social Corporativa; Transparencia; Información no financiera; Recursos Humanos; Conciliación laboral.

\section{ABSTRACT}

Directive 2014/95/EU, applicable to larger companies, requires the preparation of a Non-Financial Information Statement explaining the policies applied in relation to social aspects and the measures adopted including, among others, those relating to work-life balance as part of working conditions. This qualitative exploratory study of cases analyses the information disclosed by four companies considered sensitive to the issue of work-life balance in the first year of enforcement of RDL 18/2017 in Spain. Also carries out a selection of good disclosure practices that may serve as a guide for other companies that wish to apply it, even if they are not required to. The results confirm the positive effect of the implementation of new regulations on the level of information disclosed with respect to the previous year, but suggest that there is room for improvement in the quantity and quality of information on work-life balance disclosed by companies.

Keywords: Corporate Social Responsibility; Transparency; Non-financial Information; Human Resources; Work-life balancing.

* Correspondencia a: Ainhoa Saitua-Iribar. Facultad de Economía y Empresa (Sarriko), Avenida Lehendakari Agirre, 83, 48015-Bilbao (España) - ainhoa.saitua@ehu.eus - https://orcid.org/0000-0002-6937-1753

Cómo citar: Teixeira-Silva, Kamilla; Saitua-lribar, Ainhoa. (2020). «Nueva normativa de divulgación de información no financiera para grandes empresas. Análisis de casos sobre políticas de conciliación de la vida laboral, personal y familiar)"; Lan Harremanak, 43, 95-119. (https://doi.org/10.1387/lan-harremanak.21246).

Recibido: 20 noviembre, 2019; aceptado: 29 mayo, 2020.

ISSN 1575-7048 - elSSN 2444-5819 / (c) 2020 UPV/EHU

(c) (i) Esta obra está bajo una licencia

Creative Commons Atribución 4.0 Internacional 


\section{Introducción}

La sociedad, cada vez más preocupada por conocer sobre la gestión empresarial responsable, reclama información ya que las personas inversoras, trabajadoras, consumidoras, etc. consideran necesario disponer de información relevante sobre la gestión de las entidades en las que invierten, trabajan, consumen, etc. (ORSC, 2016). La transparencia conduce a una mejor gestión de las empresas, y no solo en relación con la información en materia financiera, sino también en relación con materias sociales y medioambientales. En este sentido, la Unión Europea ha trabajado activamente en las últimas décadas, hasta la aprobación de la Directiva 2014/95/UE de información no financiera, también conocida como Directiva de Reporting RSC, de aplicación para las empresas más grandes (Larrinaga y Luque, 2017; García, Martín y Rodríguez, 2020).

El objetivo de esta directiva es aumentar la transparencia y el rendimiento de las empresas en materia social y, por lo tanto, gestionar de manera más eficiente los riesgos y oportunidades de carácter no financiero y así contribuir con el crecimiento del empleo y la economía. Una de las novedades de esta directiva consiste en que en los informes de gestión de las empresas de un cierto tamaño se exige información sobre las políticas de diversidad y datos específicos en relación con su personal, incluido el personal directivo. Así, no se trata solo de pedir indicadores, sino que se requiere la explicación de las políticas aplicadas en relación con la cuestión y las medidas adoptadas, por lo que las empresas a las que les sea de aplicación deben hacer un análisis de los aspectos sociales (Miller, 2016).

La Directiva de Reporting fijaba un plazo de dos años para incorporarse al derecho nacional de cada estado miembro. En Espańa actualmente es de aplicación Ley 11/2018, en materia de información no financiera y diversidad. Pero inicialmente se aprobó el Real Decreto-ley 18/2017, de 24 de noviembre, por el que se modifican el Código de Comercio, el texto refundido de la Ley de Sociedades de Capital aprobado por el Real Decreto Legislativo 1/2010, y la Ley 22/2015 de Auditoría de Cuentas, en materia de información no financiera y diversidad. La modificación introducida era de aplicación a los ejercicios económicos iniciados a partir del 1 de enero de 2017. De ahí que, para el análisis de la información divulgada por las empresas, hayamos seleccionado el primer ejercicio de entrada en vigor y el inmediatamente anterior, a fin de poder comparar los cambios producidos tras la implantación de la Directiva. En cuanto a los temas sociales y relativos al personal que se deben difundir, la información hará referencia, entre otros, a las medidas adoptadas para asegurar la igualdad de género y condiciones de trabajo (Alba, 2017). En concreto, lograr una igualdad de género efectiva es el número cinco de los 17 Objetivos de Desarrollo Sostenible (ODS) de las Naciones Unidas que se aprobaron en 2015, objetivo que debe promoverse también en el ámbito empresarial y cuya divulgación no se ha investigado suficientemente aún (Saitua, 2017). 
Con estos antecedentes, este artículo es el primero que partiendo del marco teórico y normativo relativo a la divulgación de información no financiera sobre conciliación laboral, analiza la información de cuatro empresas que pueden considerarse especialmente sensibles en este tema (dos por ser reconocidas con el premio Euskadi Familia, y otras dos, como Top Employers) para los ejercicios 2016 y 2017. El objetivo principal consiste en valorar la cantidad y el nivel de calidad que proporcionan en el primer ejercicio de aplicación de la incorporación de la directiva europea a la normativa mercantil española, así como realizar una selección de las que se puedan considerar como buenas prácticas de divulgación, a fin de que puedan servir de ejemplo para otras empresas. Tras esta introducción, el apartado dos revisa brevemente la literatura académica y normativa sobre el tema, y el tercer apartado muestra la metodología empleada junto con los resultados obtenidos. El apartado cuarto se dedica a las conclusiones y reflexiones finales.

\section{Marco teórico-normativo relativo a las políticas de conciliación laboral y su divulgación como parte de la RSC}

\subsection{Las políticas de conciliación laboral como parte de la Responsabilidad Social}

Friedan (1983) denominó «doble jornada» a la realización de un trabajo remunerado y otro de tareas domésticas, fenómeno que tradicionalmente ha afectado más a las mujeres debido a la ausencia de los hombres en las tareas domésticas y de cuidado. Esto se puede constatar aún hoy en día pues más del 90\% de las excedencias que se solicitan para el cuidado corresponden a mujeres (Rodríguez, 2018). Pero el concepto de corresponsabilidad familiar — que supone la articulación de tareas "productivas» y "reproductivas» desde una perspectiva que armonice los espacios de familia y trabajo de una forma más equitativa entre hombres y mujeres - es un elemento clave para el desarrollo social de los países (Gómez y Jiménez, 2015). Últimamente, desde el mundo empresarial ha surgido incluso el movimiento Baby Friendly Companies para distinguir a las empresas que cuidan y apoyan a los empleados que deciden tener familia (RRHHDigital, 2019).

En todo caso, este debate de la conciliación forma parte de un tema más amplio sobre la Responsabilidad Social Corporativa (RSC), como herramienta que sirva para amortiguar el impacto negativo de la actividad de las empresas sobre los derechos sociales, laborales, el medioambiente y, en definitiva, sobre los Derechos Humanos. Las empresas están obligadas a respetar y aplicar la legislación laboral vigente en cada momento, pero también están capacitadas para ampliar y mejorar las condiciones establecidas. En este sentido, se puede con- 
siderar la conciliación como parte de las acciones de la Responsabilidad Social Corporativa. Las empresas pueden impulsarla por diversos motivos, pero el primordial debería ser ofrecer mejores condiciones laborales a sus empleados/as (Aranzadi, 1976; Arderiu, 1980). La esencia de la Responsabilidad consiste en la existencia de libertad y de voluntariedad, toda vez que somos responsables porque somos libres y actuamos por propia voluntad (Aranguren, 1979).

Los antecedentes de la Responsabilidad social se remontan al siglo XIx, en el marco del cooperativismo y el asociacionismo, que buscaban unir eficacia empresarial con los principios sociales de democracia, apoyo a la comunidad y justicia distributiva. Actualmente, sus máximos exponentes son las empresas de economía social, por definición empresas socialmente responsables, que implica que actúen conciliando los intereses del negocio con las expectativas que tiene la sociedad (Perdiguero, 2003; Rodríguez y Abreu, 2009). Y entendemos la responsabilidad social corporativa (RSC) como el conjunto de obligaciones y compromisos, legales y éticos, nacionales e internacionales, con los grupos de interés, que se derivan de los impactos que la actividad y operaciones de las organizaciones producen en el ámbito social, laboral, medioambiental y de los derechos humanos (De la Cuesta y Valor, 2003). Por tanto, la RSC afecta a la gestión de las organizaciones, tanto en sus actividades productivas y comerciales, como en las relaciones con sus grupos de interés, que puede mejorar mediante el nuevo estilo de relaciones «ner» (Álvarez y Apellaniz, 2017).

Los cinco principios generales que rigen la RSC son (ORSC, 2016):

— El cumplimiento de la legislación nacional vigente en cada momento.

- Su carácter global y transversal.

- Compromisos éticos y coherentes para quienes lo contraen.

- Gestión de impactos sobre la sociedad, economía y medio ambiente.

- Se dirige a la satisfacción de las expectativas y necesidades de a quién va dirigida.

Además, existen diversas iniciativas nacionales e internacionales que impulsan y/o regulan la responsabilidad social (cuadro 1).

En la actualidad, el desempeño de un trabajo, independientemente de la profesión de que se trate, se ve influenciado por la globalización de la economía, las nuevas tecnologías, y las innovaciones en las relaciones y organizaciones sociales, que están haciendo que las formas de trabajar cambien. Ello aumenta la importancia la conciliación, ya no solo como una cuestión de género, que puede tener impacto en los resultados de las empresas (Díaz, 2007), sino en el sentido de que haya mayor flexibilidad en el ámbito de las relaciones laborales. No se trata de que solo el/la trabajador/a debe adaptarse a la filosofía de la empresa, sino que la organización también debe adaptarse a las necesidades de sus miembros. 


\section{Cuadro 1}

Normativa y recomendaciones para la Responsabilidad Social corporativa

\begin{tabular}{|c|}
\hline Internacional \\
\hline $\begin{array}{l}\text { - Declaración tripartita de la OIT (2006). } \\
\text { — Global Compact o Pacto Mundial de Naciones Unidas. } \\
\text { — Global Reporting Initiative (GRI) (Iniciativa para la Rendición de Cuentas Glo- } \\
\text { bal). } \\
\text { — Líneas directrices de la OCDE para empresas multinacionales. } \\
\text { — World Business Council for sustainable Development (WBCSD). }\end{array}$ \\
\hline Europea \\
\hline $\begin{array}{l}\text { - Libro Verde «Fomentar un marco europeo para la Responsabilidad social de las } \\
\text { empresas» (UE, 2001). } \\
\text { - Norma ISO 26000:2010. } \\
\text { - Directiva 2014/95/UE. }\end{array}$ \\
\hline Española \\
\hline $\begin{array}{l}\text { - Libro Blanco de la RSE (2006). } \\
\text { - La RSE. Diálogo Social. } \\
\text { — Sistema de Gestión Ética y Socialmente Responsable SGE 21:2005. } \\
\text { - Real Decreto 221/2008, de } 15 \text { de febrero, por el que se crea y regula el Consejo } \\
\text { Estatal de Responsabilidad Social de las Empresas. } \\
\text { - Ley 2/2011, de } 4 \text { de marzo, de Economía Sostenible. } \\
\text { - Estrategia Española de Responsabilidad de las Empresas 2014/2020 (MESS, 2014). } \\
\text { - Plan de Acción Nacional de empresas y derechos humanos del } 2017 \text { (Gobierno de } \\
\text { Espańa, 2017). } \\
\text { - Ley 11/2018 que transpone la Directiva europea de información no financiera } \\
\text { UE/2014/95. }\end{array}$ \\
\hline
\end{tabular}

Fuente: elaboración propia.

El término conciliación, con el significado que se le da en el ámbito laboral, aparece por primera vez en 1974 (Stratigaki, 2004 en Alcañiz, 2015) en el articulado del Community Social Action Programme: «para asegurarse de que las responsabilidades familiares de todos los interesados puedan conciliarse con sus aspiraciones laborales». Actualmente, conciliar la vida laboral y la vida familiar se entenderá como «la participación equilibrada entre mujeres y hombres en la vida familiar y en el mercado de trabajo, conseguida a través de la reestructuración y reorganización de los sistemas, laboral, educativo y de recursos sociales, con el fin de introducir la igualdad de oportunidades en el empleo, variar los roles y estereotipos tradicionales, y cubrir las necesidades de atención y cuidado a 
personas dependientes» (Programa Óptima, s.f; Departamento de empleo y políticas sociales, s.f.).

Y relacionados con la implantación de políticas de conciliación y de otras prácticas socialmente responsables, se podrán utilizar algunos de estos instrumentos (Fabregat, 2009; Fernández, 2010; Ruiz, 2015; Leiva y Comelin, 2015; y Castro y Álvarez, s.f.), que serán parte de la organización interna de cada empresa y tendrán una integración total: Código de ética y conducta; Código de buenas prácticas laborales; Planes de igualdad; Informes de responsabilidad o memorias de sostenibilidad, etc. Incluso se deberá atender a la perspectiva de género en el lenguaje ya que no es sexista la lengua, sino su uso (Grijelmo, 2018).

En todo caso, implantar políticas de conciliación trae diversos beneficios (cuadro 2), ya sea para quien las disfruta como para quien las implanta (Izquierdo, 2017).

\section{Cuadro 2}

Posibles beneficios de la implantación de la conciliación de la vida laboral, personal y familiar

\begin{tabular}{|c|c|}
\hline $\begin{array}{l}\text { Calidad } \\
\text { en la gestión } \\
\text { de los recursos } \\
\text { humanos }\end{array}$ & $\begin{array}{l}\text { Mejora del clima laboral. } \\
\text { Mejora del estado de ánimo de los/as trabajadores/as. } \\
\text { Menor estrés y conflictos laborales. } \\
\text { Mayor satisfacción laboral. }\end{array}$ \\
\hline $\begin{array}{l}\text { Ahorro } \\
\text { económico }\end{array}$ & $\begin{array}{l}\text { Disminución de los costes de reclutamiento y plantilla. } \\
\text { Estabilidad de la plantilla = Menos gastos de contratación y for- } \\
\text { mación. }\end{array}$ \\
\hline $\begin{array}{l}\text { Aumento de la } \\
\text { productividad y } \\
\text { competitividad }\end{array}$ & $\begin{array}{l}\text { Mayor compromiso del trabajador/a. } \\
\text { Mayor rendimiento. } \\
\text { Adquisición de los objetivos de la empresa. } \\
\text { Aumento de la Capacidad productiva. } \\
\text { Incremento del Rendimiento. } \\
\text { Menor Absentismo (optimización de los horarios). }\end{array}$ \\
\hline $\begin{array}{l}\text { Mejora } \\
\text { de la imagen } \\
\text { de la empresa }\end{array}$ & $\begin{array}{l}\text { Más retención de talento. } \\
\text { Mayor número de personas candidatas. } \\
\text { Ventaja competitiva. }\end{array}$ \\
\hline
\end{tabular}

Fuente: elaboración propia basado en Izquierdo (2017). 
Debido a la dificultad que sufre el seno de la unidad familiar para que se produzca la integración plena en el mercado de trabajo, las diferentes instituciones se han visto obligadas a adoptar medidas precisas para garantizar la compatibilidad del trabajo y la familia (Rodríguez, 2008). En el marco de la Unión Europea, las políticas activas de empleo buscan contribuir a la inclusión de la conciliación de la vida laboral y familiar, relacionada con la igualdad de oportunidades entre mujeres y hombres. Así, se ha producido la incorporación expresa de esta temática en el cuarto pilar de las directrices comunitarias en materia de empleo, que ha facilitado su inclusión en la agenda de la negociación colectiva de los países miembros, incluida España (Martin y Carrasquer, 2005). Sin embargo, las políticas públicas para la conciliación familia-trabajo se tornan insuficientes si se entiende que tal conciliación debe resolverse centrada en facilitar las tareas a las mujeres, desplazando la corresponsabilidad (Leiva y Comelin, 2015).

Las políticas familiarmente responsables habitualmente son llamadas «medidas o políticas de conciliación" y se pueden definir como aquellas prácticas empresariales formales que ayudan a las personas empleadas a equilibrar la relación entre su trabajo y la vida familiar. Estas no son un conjunto homogéneo de actuaciones, pero tienen en común que pretenden conciliar la esfera productiva y el ámbito de la vida privada, particularmente el aspecto familiar, mediante actuaciones empresariales que van más allá de las legalmente establecidas por las normas (EOI, 2006). Todo esto juega un papel muy importante, ya que una vez las empresas conocen los problemas de sus empleados/as, a través de los diferentes instrumentos y métodos mencionados puedan darles una solución. Los diferentes instrumentos disponibles para hacer frente a la cuestión de la conciliación laboral, pueden constituir una estrategia empresarial que ayuda a una mejor gestión de los recursos humanos y del entorno social de la empresa mediante la mejora de la relación con sus empleados/as.

\subsection{Iniciativas públicas para la promoción y regulación de la conciliación laboral}

Desde 1919 que se comenzó con la regulación internacional para la protección de la mujer embarazada, hasta el día de hoy, ha habido numerosas normativas (cuadro 3) que han intentado dar solución al problema de la conciliación laboral (Orellana, 2002; Molina, 2004).

La Ley 39/1999 introdujo el concepto moderno de conciliación entre la vida familiar y laboral en el ordenamiento jurídico español (Menéndez, 2018). Cierto es que el derecho del trabajo ya había tratado este tema, pero desde la perspectiva de la protección de la mujer embarazada, sin tener en cuenta el enfoque del género (Rodríguez, 2008). 


\section{Cuadro 3}

\section{Marco normativo de la conciliación laboral, personal y familiar}

\section{Europeo}

- Convenio OIT n. ${ }^{\circ} 103$ de 1952.

- Carta Social Europea de 18 de octubre de 1961.

- Convenio OIT n. ${ }^{\circ} 156$ de 1981.

- Carta Comunitaria de Derechos Sociales Fundamentales de 19 de diciembre 1989.

- Carta de los Derechos fundamentales de la Unión europea, de 7 de diciembre de 2000.

\section{Español}

— Ley 39/1999, de 5 de noviembre para promover la conciliación de la vida familiar y laboral de las personas trabajadoras.

— Ley Orgánica 3/2007, de 22 de marzo, para la igualdad efectiva de mujeres y hombres.

— Real Decreto-Ley 3/2012, de 10 de febrero, de medidas urgentes para la reforma del mercado laboral.

- Real Decreto Legislativo 2/2015, de 23 de octubre, por el que se aprueba el texto refundido de la Ley de Estatuto de los Trabajadores.

- Real Decreto Legislativo 8/2015, de 30 de octubre, por el que se aprueba el texto refundido de la Ley General de la Seguridad Social.

\section{Autonómico (País Vasco)}

— Ley 13/2008 de 12 de diciembre, de apoyo a las Familias.

— Decreto 177/2010 de 29 de junio, sobre ayudas para la conciliación de la vida familiar y laboral.

— Decreto 31/2015 de 17 de marzo, de modificación del Decreto sobre ayudas para la conciliación de la vida familiar y laboral.

Fuente: elaboración propia.

Por su parte, la Ley Orgánica 3/2007, también llamada Ley de Igualdad, en el artículo 1 establece que «Las mujeres y los hombres son iguales en dignidad humana, e iguales en derechos y deberes. Esta Ley tiene por objeto hacer efectivo el derecho de igualdad de trato y de oportunidades entre mujeres y hombres, en particular mediante la eliminación de la discriminación de la mujer, sea cual fuere su circunstancia o condición, en cualesquiera de los ámbitos de la vida y, singularmente, en las esferas política, civil, laboral, económica, social y cultural para, en el desarrollo de los artículos 9.2 y 14 de la Constitución, alcanzar una sociedad más democrática, más justa y más solidaria».

La Ley Orgánica 3/2007, para la Igualdad Efectiva de Mujeres y Hombres, se inspira en parte en la Ley Orgánica 1/2004, de Medidas de Protección Integral contra la Violencia de Género, a partir de la cual se trata de evitar que los 
mecanismos jurídicos de conciliación de la vida laboral y familiar repercutan negativamente en sus titulares, que son tradicionalmente, las mujeres (Rodríguez, 2008). Por último, el Real Decreto Legislativo 8/2015 dispone de varios capítulos dedicados a la protección de la familia, estableciendo diversas prestaciones y situaciones protegidas que son de aplicación a todos los/as trabajadores/as.

Si bien existen diversas medidas públicas para hacer frente a la conciliación, tomaremos como referencia la Guia de ayudas sociales y servicios para las Familias (MSCBS, 2018), que publica anualmente el Ministerio de Sanidad, Seguridad Social e Igualdad. Y mencionamos las prestaciones de carácter retributivo que generan las personas trabajadoras (capítulos VI, VII, VIII, IX y X de la LGSS):

i. Maternidad (arts. 177-182 LGSS) (art. 48.4, 5,6 ET).

ii. Paternidad (arts. 183-185) (48.7 ET).

iii. Riesgo durante el embarazo (arts. 188-189 LGSS).

iv. Riesgo durante la lactancia (arts. 188-189 LGSS).

v. Cuidado de menores con enfermedades graves (190 LGSS) (37.6 ET).

vi. Reducción de jornada (37.4, 5, 6,7 y 8 ET).

vii. Excedencias (art. 46 ET).

Además de éstas de carácter público, también existe una serie de medidas que pueden adoptar las empresas para facilitar la conciliación de la vida laboral, familiar y personal que, si bien no son de obligatorio cumplimiento, se deberían tener en cuenta para el mejor funcionamiento de las entidades (García, 2017).

a) Mejorar los términos establecidos en la normativa. Por ejemplo, pueden ampliar los permisos legales retribuidos.

b) Horarios flexibles de entrada y salida. Cómo distribuir la jornada, posibilidad de ajustar los horarios, facilitar el teletrabajo, etc.

c) Posibilidad de compatibilizar las vacaciones, dando la opción de que los/as progenitores pasen más tiempo con sus hijos/as y no tengan que recurrir a otros/as familiares para que se hagan cargo de los/as menores.

d) Servicios de guardería.

e) Comedores: con el fin de reducir el tiempo de traslado, además del tiempo de comida, para poder salir antes del trabajo y favorecer así la conciliación.

f) Gimnasio. Solo un 7\% de las empresas tienen convenio con centros deportivos para que así sus trabajadores dispongan de descuentos, según una encuesta realizada por el instituto andaluz de deporte (García, 2018). El estudio de Musich, Hook, Baaner y Edington (2006) concluía que promover la buena salud en el ámbito laboral disminuye el absentismo de las personas trabajadoras y minimiza la pérdida de productividad (García, 2017). 
g) Cultura empresarial. Se trata de medidas que se asientan en las políticas de recursos humanos de las empresas, es decir, de regulación interna.

En todo caso, a modo de reconocimiento externo, el certificado EFR (Empresas Familiarmente Responsables), promovido por la Fundación Másfamilia y apoyado por el Ministerio de Sanidad y Política Social, reconoce a aquellas organizaciones que se implican en la generación de una nueva cultura del trabajo, incorporando un sistema integral de gestión, que permita una eficaz armonía entre la esfera laboral y la familiar y personal, basada en la flexibilidad, el respeto y el compromiso mutuos.

\subsection{Divulgación de información no financiera}

Una vez que las empresas llevan a cabo políticas sociales, pueden divulgar sobre ello. Esto puede ocurrir de forma obligatoria, en aplicación primero del RDL 18/2017 y, posteriormente de la Ley 11/2018, para las sociedades de capital que concurran los siguientes requisitos: a) que el número medio de personas trabajadoras empleadas durante el ejercicio sea superior a 500 (este límite se reducirá a 250 a partir de 2021, obligando así a una mayor cantidad de empresas); y b) que o bien tengan la consideración de entidades de interés público de conformidad con la legislación de auditoría de cuentas, o bien, durante dos ejercicios consecutivos reúnan, a la fecha de cierre de cada uno de ellos, al menos dos de las circunstancias siguientes: 1) que el total de las partidas del activo sea superior a 20 millones de euros; 2) que el importe neto de la cifra anual de negocios supere los 40 millones de euros; y 3) que el número medio de personas trabajadoras empleadas durante el ejercicio superior a 250.

En lo que se refiere a contenidos, la Ley 11/2008 obliga a informar sobre las políticas aplicadas por la empresa y los resultados obtenidos como consecuencia de dichas políticas, en cuestiones relativas a organización del trabajo, salud laboral, diversidad de género, compromiso con los Objetivos de Desarrollo Sostenible (ODS), etc.

Y en cuanto a cómo debe informarse, esta modificación del artículo 49 del Código de Comercio aclara que los indicadores clave de resultados no financieros que se divulguen, deberán ser adecuados a las actividades de la empresa, de acuerdo con parámetros de gestión y evaluación. Así, es la empresa quien decide sobre qué aspectos concretos informará, mediante qué indicadores, etc., de modo que no está garantizada la comparabilidad en el tiempo ni entre empresas, poniéndose en cuestión la utilidad de la información divulgada debido a la falta de comparabilidad porque existen muchas guías y aún es necesario avanzar en normalización y supervisión (IFAC, 2020).

En todo caso, según la Ley 11/2008, los indicadores divulgados deben cumplir con los criterios de comparabilidad, materialidad, relevancia y fiabilidad, de forma que la información debe ser precisa, comparable y verificable. 
En este sentido, en Espańa, el modelo o guía más utilizado para la elaboración de dicha información es el recomendado por la Global Reporting Initiative (GRI) (Ibercampus, 2017; García et al., 2020), organización que en los últimos años está emitiendo recomendaciones para incorporar la información específica sobre los ODS en la información divulgada por las empresas (GRI 2018, 2020).

Por último, también puede informarse de forma voluntaria aun cuando no se cumplan los límites de tamaño marcados por la ley (AECA, 2016), sabiendo además que, si bien la Ley 11/2018 se introdujo para las cuentas anuales del ejercicio 2018 y posteriores, a partir del ejercicio 2021 abarcará a un mayor número de empresas que deberán ir preparándose para cuando les resulte obligatorio.

\section{Análisis de las prácticas de divulgación sobre conciliación laboral, personal y familiar y selección de las mejores prácticas}

\subsection{Metodología}

Una vez revisados los principales conceptos teóricos y normativos, podemos señalar la importancia de la conciliación de la vida laboral, personal y familiar tanto para las personas trabajadoras como para las propias entidades, y cómo se ha convertido en un factor importante dentro de la responsabilidad corporativa que puede ser divulgada por las empresas. En este sentido, la información divulgada por las empresas puede considerase como una señal si refleja información sobre características no observables de las empresas y de su actividad, pero no sólo la información en sí misma puede tener ese carácter, sino también la forma de darla, los cambios, o incluso la ausencia de información (Giner, 1995). Además, el impacto de las nuevas regulaciones que puedan ponerse en marcha quedará parcialmente mitigado por su tradicional tendencia al bajo nivel de divulgación (Giner, 1995).

Así, el objetivo principal de esta parte del trabajo es conocer las prácticas de gestión que se llevan a cabo en las empresas y qué información divulgan sobre todo ello. Además, se pretende identificar los cambios producidos por la aplicación del RDL 18/2017, de 24 de noviembre, en materia de información no financiera y diversidad, y cómo ha influido en la información disponible. El segundo objetivo consiste en seleccionar las que se puedan considerar como mejores prácticas de divulgación sobre el tema analizado para que puedan servir de guía a otras empresas. Para llevar a cabo este trabajo, nos hemos basado en la metodología utilizada por la literatura anterior (Giner, 1995; Aranguren y Ochoa, 2008; García, Prado y Frías, 2013; Saitua et al., 2014a, 2014b, 2015).

En nuestro caso, la información analizada se considera obligatoria en la medida en que las empresas están obligadas a presentar el Estado de Información 
No Financiera debido a que cumplen con los criterios de tamaño establecidos en la Ley. Y además, el tema sobre Conciliación laboral analizado es uno de los aspectos que cita expresamente la ley, por lo que se puede considerar contenido obligatorio. Sin embargo, en la medida en que la profundidad o nivel de desarrollo de dicha información, así como los indicadores que pudiera eventualmente divulgar cada empresa quedan a discreción de cada entidad, podemos decir que el análisis de las diferentes prácticas de divulgación dará como resultado la evidencia de los distintos niveles de compromiso voluntario para con los criterios de relevancia, fiabilidad y comparabilidad requeridos por la ley.

La selección de las empresas para el análisis de casos, se realizó tomando como referencia, por un lado, dos empresas vascas que habían ganado el Premio Familia Euskadi 2016/2017, de diferentes sectores de actividad: Iberdrola de suministro de energía eléctrica, y Euskaltel de información y comunicaciones. Por otro lado, se buscó información disponible sobre empresas incluidas en el Top Employers España (Compromiso Empresarial, 2018). En este caso se han seleccionado Banco Santander y Bankinter, que pertenecen ambas al sector financiero. A fin de determinar el periodo de tiempo se ha partido del ejercicio 2016 y se comparará con el 2017, basándonos en la transposición de la Directiva 2014/95/CE de Información no financiera, que una vez puesta en vigor en España se empezaría a aplicar a los ejercicios económicos iniciados a partir del 1 de enero del 2017. Esto permitirá observar si se han producido cambios en el contenido de la información analizada. Para la recogida y análisis de los datos obtenidos, se han analizado los informes anuales de cada empresa en los respectivos años. En concreto, el Informe de Gestión consolidado, lo que nos llevará a obtener un total de ocho observaciones empresa/año.

La técnica utilizada ha sido el análisis del contenido informativo, en el que se toma como unidad un componente de comunicación identificable a través del que se miden las variables representativas de las prácticas divulgativas, técnica apropiada, en particular, cuando el lenguaje del sujeto es crucial para la investigación (Holsti, 1969). Este proceso requiere la definición de ítems homogéneos de datos y agrupación en los mismos de la información de las memorias publicadas por las empresas (García, Prado y Frías, 2013). Una vez establecidos los ítems, se establece la medición del nivel de información divulgada para cada uno de ellos. Una posibilidad consiste en utilizar programas informáticos avanzados que extraen información de los informes analizados (Gamerschlag et al., 2011) pero la codificación manual puede resultar más adecuada en algunos casos (Guillamón, 2011), debido a la mayor capacidad de la persona que realiza la codificación manual para comprender y valorar el contexto o significado específico de cada texto.

Dependiendo de la unidad de análisis elegida, hay diversas formas de aplicar el análisis de contenido, por ejemplo, contando palabras, frases o sec- 
ciones, o bien leyendo todo el texto (Neuendorf, 2002). El debate sobre cuál debería ser la unidad de análisis a utilizar en el análisis de contenido se centra en cuál es el medio más efectivo para inferir la intención de divulgar a partir de datos volumétricos (Gray et al., 1995; Milne y Adler, 1999; Beattie et al., 2004; Campbell, 2004; Ax y Marton, 2008). La ventaja de utilizar las palabras como unidad se centraría en que el codificador no necesita aplicar juicios subjetivos, de modo que resulta un método fiable en el sentido de que se obtienen los mismos resultados en repetidas ocasiones, resultando fácilmente replicable (Gamerschlag et al., 2011). Para nuestro estudio, medimos el número de párrafos y de tablas que incluyen determinadas palabras, lo que nos permitirá obtener un ranking de las empresas que más información divulgan al respecto.

Para realizar la selección de la información dentro del Informe de Gestión, se ha filtrado a través de los términos Conciliación, Familia, Mujer, Maternidad, Paternidad, Permisos y Flexibilidad. Aquí tomamos como referencia dos unidades para poder observar cuál de las cuatro empresas las menciona más: el número de veces que aparece la palabra conciliación y el número de veces que aparece la palabra maternidad y/o paternidad. Por otro lado, los diferentes temas que se han utilizado para realizar el análisis de contenido están basados en las cuestiones desarrolladas a lo largo del marco teórico-normativo: 1) Datos sobre la plantilla, 2) Políticas de igualdad, 3) Políticas de conciliación, 4) Planes de actuación, 5) Comité de conciliación, 6) Existencia de manuales específicos de conciliación, 7) Datos sobre permisos 8) Datos sobre absentismo, 9) Posibilidad de horarios flexibles, 10) Compatibilización de vacaciones, 11) Disponibilidad de guardería, 12) Comedores en los centros de trabajo. 13) Disponibilidad de gimnasios, 14) Teletrabajo.

Si miramos a la naturaleza de las informaciones, Beretta et al. (2007) distinguen divulgaciones soft (cualitativas/no verificables) y hard (cuantitativas/ verificables), encontrando que la información hard es más efectiva, tanto en la mejora (acierto) de los pronósticos, como reduciendo la dispersión de los pronósticos de analistas de inversión. Así, para medir la información recogida en los Informes de Gestión, al estilo de Aranguren y Ochoa (2008), se ha optado por codificar la información mediante estos tres valores: 0 No divulga información, 1 Información meramente cualitativa, y 2 Información cualitativa y cuantitativa.

Finalmente, basándonos en la literatura anterior sobre la escasa divulgación informativa de las empresas españolas y el limitado alcance de las nuevas normativas (Giner, 1995), podemos establecer las siguientes preguntas de investigación. En primer lugar, debido a que la información no financiera que las empresas están obligadas a difundir en relación con temas sociales y personal puede ser de diversa índole, planteamos P1: ¿La cantidad y calidad de información divul- 
gada sobre conciliación en el Informe de Gestión en general es escasa? Por otro lado, como hemos dicho, la trasposición de la Directiva 2014/95/CE de Información no financiera mediante el Real Decreto-ley 18/2017 es aplicable para los ejercicios económicos iniciados a partir del 1 de enero de 2017 de modo que P2: ¿La cantidad de información sobre conciliación en el Informe de Gestión aumenta de 2016 a 2017? Por último, debido a que los Premios Familia son concedidos atendiendo a diversos factores y que entre ellos podemos encontrar la "Conciliación familia-trabajo", P3: ¿La cantidad divulgada por las empresas ganadoras del Premio Familia Euskadi es mayor que la del resto?

\subsection{Resultados del análisis}

Si bien la metodología principal del estudio es cualitativa, para poder realizar comparaciones entre las empresas objeto de análisis, a continuación se extraen algunos datos cuantitativos que se han venido utilizando en la literatura académica sobre divulgación como indicativos de la calidad de la información divulgada.

\section{a) Análisis cuantitativo del número de párrafos, tablas y palabras}

Los resultados muestran que Iberdrola y Euskaltel superan ampliamente a las demás empresas en el número párrafos y tablas.

Tabla 1

Ranking de empresas en función del número de párrafos y tablas sobre conciliación en 2017

\begin{tabular}{l|rrr|rrr|rrr|rrr}
\hline & \multicolumn{2}{|c|}{ Iberdrola } & \multicolumn{2}{c|}{ Euskaltel } & \multicolumn{3}{c|}{ Bankinter } & \multicolumn{2}{c}{ Santander } \\
\cline { 2 - 11 } & 2016 & 2017 & Var $\%$ & 2016 & 2017 & Var\% & 2016 & 2017 & Var\% & 2016 & 2017 & Var\% \\
\hline N. ${ }^{o}$ de párrafos & 2 & 11 & $450 \%$ & 4 & 6 & $50 \%$ & 1 & 4 & $300 \%$ & 3 & 1 & $-67 \%$ \\
N. ${ }^{o}$ de tablas & 0 & 1 & $100 \%$ & 0 & 1 & $100 \%$ & 0 & 0 & $0 \%$ & 0 & 0 & $0 \%$ \\
\hline
\end{tabular}

Fuente: elaboración propia.

Además, resultan ser las que han ganado el Premio Familia Euskadi, lo que confirmaría la pregunta de investigación $P 3$ por la que suponíamos que debido a esta circunstancia encontraríamos un mayor volumen de información divulgada.

En cuanto al número de ocasiones en las que se citan los términos de conciliación o de maternidad/paternidad, si tomamos el total de observaciones, las empresas ocupan el mismo lugar en el ranking (tabla 2), si bien las variaciones no han ido en la misma proporción para cada empresa. 
Tabla 2

Número de veces que aparece el término analizado por empresa y año

\begin{tabular}{|c|c|c|c|c|c|c|c|c|c|c|c|c|}
\hline & \multicolumn{3}{|c|}{ Iberdrola } & \multicolumn{3}{|c|}{ Euskaltel } & \multicolumn{3}{|c|}{ Bankinter } & \multicolumn{3}{|c|}{ Santander } \\
\hline & 2016 & 2017 & $\operatorname{Var} \%$ & 2016 & 2017 & $\mathrm{Var} \%$ & 2016 & 2017 & $\operatorname{Var} \%$ & 2016 & 2017 & $\operatorname{Var} \%$ \\
\hline Conciliación & 2 & 19 & $850 \%$ & 2 & 3 & $50 \%$ & 1 & 2 & $100 \%$ & 2 & 1 & $-50 \%$ \\
\hline Mater/pater & 0 & 12 & $100 \%$ & 0 & 1 & $100 \%$ & 0 & 0 & $0 \%$ & 0 & 0 & $0 \%$ \\
\hline TOTAL de observaciones & \multicolumn{3}{|c|}{33} & \multicolumn{3}{|c|}{6} & \multicolumn{3}{|c|}{3} & \multicolumn{3}{|c|}{3} \\
\hline Variación media & \multicolumn{3}{|c|}{$475 \%$} & \multicolumn{3}{|c|}{$75 \%$} & \multicolumn{3}{|c|}{$50 \%$} & \multicolumn{3}{|c|}{$-25 \%$} \\
\hline
\end{tabular}

Fuente: elaboración propia.

El aumento más significativo se produce en Iberdrola, siendo ésta la que más observaciones presenta en total (33), mientras que Euskaltel y Bankinter muestran un aumento muy ligero pues se trata de pocas observaciones. Por el contrario, Santander es la única empresa que presenta una variación negativa.

\section{b) Análisis de contenido temático}

En 2016, las empresas que más información difunden son Euskaltel y Santander (puntuación total = 7). En cambio, en 2017 son Iberdrola (12) y Euskaltel (10). Así, la empresa que más información comunica en 2017 es Iberdrola, que aporta información sobre medidas, algunas de ellas bastante innovadoras y además proporciona datos cuantitativos. Iberdrola también es la única empresa que revela disponer de un "Manual específico para la Conciliación». Esta información se encuentra en el Informe de Gestión de 2017 donde explica que ofrece diversas medidas a sus empleados/as, como pueden ser la adaptación de sus jornadas a los días no lectivos, además de implantar cursos de formación para niños/as, escuela de padres/madres Iberdrola, y campamentos de verano para hijos/as de empleados/as.

El Banco Santander es la única empresa en la que se ha producido una evolución negativa, reduciendo de forma significativa la información disponible del año 2016 al 2017. En todo caso, en general, las empresas han evolucionado positivamente en el número de temas que tratan de un ańo a otro. La mayoría de los temas que se han tratado en el informe de gestión del 2016 se han mantenido en el 2017, incrementándose algunos más este último año.

Entre los temas más mencionados, destacamos los datos sobre la plantilla (puntuación total $=16$ ), las políticas de conciliación $(8)$, los planes de actuación (8) y las políticas de igualdad (8), que son los temas que las cuatro empresas más han mencionado en sus informes de gestión cuando trataban la temática de la conciliación. Pero la mayoría de estos temas tiene solamente datos cualita- 
tivos, a excepción de los datos de la plantilla, sobre los permisos y sobre los absentismos, en los que las informaciones disponibles son tanto cualitativas como cuantitativas.

Tabla 3

Ranking de temas citados en relación con la conciliación laboral

\begin{tabular}{|c|c|c|c|c|c|c|c|c|c|c|c|c|c|}
\hline \multirow{2}{*}{ Temas } & \multicolumn{3}{|c|}{ Iberdrola } & \multicolumn{3}{|c|}{ Euskaltel } & \multicolumn{3}{|c|}{ Santander } & \multicolumn{3}{|c|}{ Bankinter } & \multirow{2}{*}{ TOTAL } \\
\hline & 2016 & 2017 & Var & 2016 & 2017 & Var & 2016 & 2017 & Var & 2016 & 2017 & Var & \\
\hline Datos sobre la plantilla & 2 & 2 & 0 & 2 & 2 & 0 & 2 & 2 & 0 & 2 & 2 & 0 & 16 \\
\hline Políticas de conciliación & 1 & 1 & 0 & 1 & 1 & 0 & 1 & 1 & 0 & 1 & 1 & 0 & 8 \\
\hline Planes de actuación & 1 & 1 & 0 & 1 & 1 & 0 & 1 & 1 & 0 & 1 & 1 & 0 & 8 \\
\hline Políticas de igualdad & 1 & 1 & 0 & 1 & 1 & 0 & 1 & 1 & 0 & 1 & 1 & 0 & 8 \\
\hline Horarios flexibles & 1 & 1 & 0 & 1 & 1 & 0 & 1 & 0 & -1 & 0 & 0 & 0 & 5 \\
\hline Datos sobre los permisos & 0 & 2 & 2 & 0 & 2 & 2 & 0 & 0 & 0 & 0 & 0 & 0 & 4 \\
\hline Guarderías & 0 & 1 & 1 & 0 & 0 & 0 & 1 & 0 & -1 & 0 & 1 & 1 & 3 \\
\hline Datos sobre el absentismo & 0 & 0 & 0 & 0 & 2 & 2 & 0 & 0 & 0 & 0 & 0 & 0 & 2 \\
\hline Vacaciones & 0 & 1 & 1 & 0 & 0 & 0 & 0 & 0 & 0 & 0 & 1 & 1 & 2 \\
\hline Gimnasios & 0 & 1 & 1 & 0 & 0 & 0 & 0 & 0 & 0 & 0 & 1 & 1 & 2 \\
\hline Manuales de conciliación & 0 & 1 & 1 & 0 & 0 & 0 & 0 & 0 & 0 & 0 & 0 & 0 & 1 \\
\hline Teletrabajo & 0 & 0 & 0 & 1 & 0 & -1 & 0 & 0 & 0 & 0 & 0 & 0 & 1 \\
\hline Comité de conciliación & 0 & 0 & 0 & 0 & 0 & 0 & 0 & 0 & 0 & 0 & 0 & 0 & 0 \\
\hline Comedores & 0 & 0 & 0 & 0 & 0 & 0 & 0 & 0 & 0 & 0 & 0 & 0 & 0 \\
\hline TOTAL & 6 & 12 & 6 & 7 & 10 & 3 & 7 & 5 & -2 & 5 & 8 & 3 & 60 \\
\hline$\%$ & 100 & 200 & +100 & 100 & 142,8 & $+42,8$ & 100 & 71,4 & $-28,6$ & 100 & 160 & +60 & \\
\hline
\end{tabular}

Fuente: elaboración propia.

Una vez que verificamos que muchos temas y medidas de gran importancia no son mencionados, y que además los citados se tratan solo de forma cualitativa, y en muchos de los casos sin llegar a ser desarrollados, podemos responder afirmativamente a la pregunta $P 1$, ya que en general la información no financiera divulgada aún es escasa en cantidad y en calidad. En todo caso, el mayor número de observaciones obtenidas es del año 2017. Es decir, que la información no financiera divulgada ha aumentado de un año a otro, lo que responde afirmativamente a la pregunta P2, en el sentido de que se ha producido un aumento de la información no financiera divulgada por la mayoría de las empresas analizadas, lo que corroboraría el efecto positivo que ha producido la aprobación de la RDL 18/2017.

En cuanto a las empresas seleccionadas como posibles líderes en este tema, las incluidas en el Premio Euskadi Familia (Iberdrola y Euskaltel) destacan por 
comunicar mucha más información sobre la conciliación y de mayor calidad. Las dos empresas incluyen la conciliación de la vida laboral, personal y familiar como uno de sus valores y parte de su misión. En cambio, las empresas incluidas en el Top Employers España, presentan escasa información. Además, Bankinter tiene una evolución positiva difundiendo más información de un año a otro, pero la evolución de Santander es negativa. Por consiguiente, podemos responder positivamente a la pregunta $P 3$, donde planteábamos si la cantidad de información divulgada por las empresas de la primera categoría sería más abundante y de mejor calidad que las de la segunda.

\section{c) Selección de informaciones a destacar obtenidas en los informes de gestión}

De la información recogida a lo largo del análisis, destacamos algunos ejemplos de divulgación relativos a las políticas que disponen las organizaciones para mejorar y fomentar la conciliación laboral y personal, así como relativos a las medidas concretas que aplican en las diferentes áreas que hemos identificado en el marco teórico.

— Gestión de la sostenibilidad, los PRINCIPIOS que rigen esta política son: la gestión avanzada de las personas como principal capital del banco, fomentando su bienestar y motivación a través de medidas de conciliación, desarrollo personal y profesional, y promoción de la salud (Bankinter, 2016, pág. 697).

- Entre las políticas que Iberdrola aplica en el ámbito de sus relaciones laborales, está la Política de IGUALDAD de oportunidades y conciliación, los principales objetivos en este ámbito se han concretado en: -El fomento de la conciliación de la vida laboral y familiar de los empleados, que incluye medidas para hacer compatible la experiencia positiva de la maternidad y paternidad con una carrera profesional de éxito. -El desarrollo de relaciones laborales basadas en la igualdad de oportunidades, la no discriminación y el respeto a la diversidad (Iberdrola, 2017, pág. 1140).

- Banco Santander, en la ENCUESTA de compromiso los temas de flexibilidad y conciliación son los que más han evolucionado desde 2014: el $78 \%$ de los empleados afirman que su jefe les facilita el equilibrio entre la vida laboral y profesional (50\% en 2014) (Banco Santander, 2016, pág. 393).

—En el año 2016 «en Euskaltel ... hemos trabajado en nuevas iniciativas englobadas dentro del Plan de Conciliación. Escuchamos a las personas y damos solución a los problemas individuales que cada una de ellas nos presenta: cambio de HORARIOS por necesidades puntuales, teletrabajo durante el periodo de enfermedad de un familiar directo, traslado temporal al centro de trabajo más próximo al domicilio familiar en los meses de verano, etc.» (Euskaltel, 2016, pág. 66). 
— Familias Inteligentes: Euskaltel ha desarrollado un proyecto junto a la ONG AFAE (Acción familiar de Euskadi) en materia de conciliación, prevención de riesgos psicosociales y promoción de la salud, que incluye tres seminarios de FORMACIÓN familiar para la capacitación de las personas que constituyen sus equipos de trabajo, en la búsqueda de un desarrollo óptimo de su vida personal, familiar y laboral (Euskaltel, 2017, pág. 232).

- La creación de un lactario en el Centro Corporativo de Boadilla del Monte, como refuerzo de nuestra Política de Protección a la MATERNIDAD y como una medida más de flexibilidad que permite un mejor equilibrio entre la vida profesional y laboral, favoreciendo la lactancia materna tras la incorporación al trabajo. Aunque ya teníamos salas de lactancia en otros países, en España Banco Santander se convierte en la primera empresa en poner este servicio a disposición de sus trabajadoras (Banco Santander, 2016, pág. 395).

- En España, Iberdrola ha sido la primera empresa del Ibex 35 en aplicar la jornada continuada, una medida pionera, entre el conjunto de más de las 70 prácticas recogidas en el Manual de politicas de conciliación de la empresa, entre las que también destaca: Servicio de GUARDERÍA como medida para favorecer la conciliación (Iberdrola,2017, pág. 1141/1001).

- Bankinter tiene en marcha una serie de iniciativas para potenciar el bienestar de sus empleados tanto en su dimensión profesional como personal, como la Semana de la Salud, que incluye tanto cursos de ergonomía o nutrición como consejos para optimizar el EJERCICIO físico, y la Escuela de Verano, donde se imparten disciplinas formativas y lúdicas orientadas a promover la salud y el bienestar (Bankinter, 2017, pág. 770).

\section{d) Discusión de los resultados}

Se puede observar que las empresas analizadas sí divulgan información sobre la conciliación laboral, personal y familiar en sus informes, lo que muestra que es un tema que preocupa o que, al menos, se tiene en cuenta a la hora de realizar la gestión empresarial. Pero en el presente trabajo se ha puesto en manifiesto que, a pesar de que se haya producido un aumento después de la puesta en vigor de la Directiva 2014/95, una vez que la nueva normativa obliga a las empresas a difundir información no financiera, ésta sigue siendo escasa sobre el tema de conciliación. Así, la gran mayoría de las organizaciones solo cita algunos términos, sin llegar a desarrollar y explicar en profundidad las diferentes medidas que desarrollan dentro de las organizaciones y que son aplicables al personal.

Entendemos que algunas claves que servirían para mejorar la conciliación de la vida laboral, personal y familiar podrían ser un mayor compromiso con la igualdad entre mujeres y hombres, y con la conciliación de la vida laboral, fa- 
miliar y personal; conocer las verdaderas necesidades de las/los trabajadores/es en materia de conciliación para dar mejores soluciones a sus problemas estableciendo unas medidas adecuadas; disponer de un departamento específico para este tema; promover una cultura de responsabilidad social de éxito y que contribuya al mismo tiempo al bienestar de la sociedad; jornadas informativas para un mayor conocimiento de los recursos y medidas disponibles, y finalmente hacer públicos sus valores, desempeño, seguimiento y evolución tras la implantación de las medidas de conciliación.

Por otra parte, según nuestros resultados, los datos revelados en general son meramente cualitativos pues los datos cuantitativos son escasos y, en algunos casos, inexistentes. En conclusión, los resultados obtenidos muestran que la información divulgada por las empresas no es suficiente para conocer con exactitud las diferentes prácticas de conciliación laboral que llevan a cabo, ya que informan de la existencia de políticas, pero no describen cómo las implementan, ni qué consecuencias y resultados obtienen con ellas. Así, concluimos que la información divulgada por las empresas analizadas sobre conciliación laboral y personal es aún mejorable.

\section{Conclusiones y reflexiones finales}

En la sociedad se sigue dando desigualdad de género en el cuidado doméstico y ello provoca consecuencias, como la brecha salarial, entre otras, pero la conciliación no es un tema que afecte únicamente a la mujer, sino a toda la sociedad en su conjunto, pues todos/as los/las trabajadores/as deben tener derecho a conciliar su vida personal y laboral. En todo caso, recientemente ha surgido la obligación para las empresas de hacerse socialmente responsables e incluso de informar sobre ello en algunos casos.

Este estudio confirma el efecto positivo de la implantación de nueva normativa sobre divulgación de información no financiera en España por parte de las empresas analizadas. Sin embargo, pone de manifiesto que aún hay margen de mejora en la cantidad y calidad de la información divulgada por las empresas. La principal limitación del trabajo consiste en que, al tratarse de un análisis de casos, no puede realizarse una extrapolación generalizada de los resultados obtenidos. Futuras investigaciones podrán analizar la divulgación de otras empresas obligadas o incluso no obligadas a divulgar información sobre conciliación de la vida laboral y personal, e incluso de otros temas, en aplicación de la Ley $11 / 2018$. En todo caso, se muestran ejemplos en forma de buenas prácticas a considerar por otras empresas que estando obligadas por la Ley 11/2018 deban divulgar sobre este tema, o incluso, a empresas de menor tamańo que quieran hacerlo voluntariamente. 


\section{Bibliografía}

AECA (Asociación Española de Contabilidad y Administración de Empresas) (2016). Responsabilidad Social interna. Delimitación conceptual e información. Documento n. ${ }^{\circ} 10$ de RSC. Madrid. Disponible en: https://aeca.es > old > tienda (accedido: 27-5-2020).

Alba, Carmen (2017). «Las grandes empresas deberán informar sobre su política de anticorrupción", Elconfidencial, Disponible en: https:/www.elconfidencial.com/ empresas/2017-09-01/consejo-de-ministros-finanzas-anteproyecto_1437104/ (accedido: 27-5-2020).

AlcañIz Moscardó, Mercedes (2015). «Género con clase: la conciliación desigualdad de la vida laboral y familiar». Revista Española de Sociología, núm. 23, 29-55. Disponible en: http://repositori.uji.es/xmlui/bitstream/handle/10234/150765/66900. pdf?sequence=1 \&isAllowed $=y$ (accedido: 27-5-2020).

Alvarez-Sainz, María y Apellaniz-Valle, Kepa Xabier (2017). "Nuevo estilo de relaciones en la Sociedad del Conocimiento. La Gestión de conflictos en las organizaciones de Ner Group en el País Vasco». Revista Galega de Economía, Universidad de Santiago de Compostela. Facultad de Economía y Empresa, 26(1), 31-44. Disponible en: https://ideas.repec.org/a/sdo/regaec/v26y2017i1_3.html (accedido: 27-5-2020).

Aranguren, José Luis (1979). Ético. Ed. Alianza Universidad Textos.

Aranguren Gómez, Nagore y Ochoa Laburu, Elena (2008). «Divulgación de información sobre empleados y medio ambiente en España y Alemania: una nota de investigación». Revista de contabilidad, 11, 2, 123-142. Disponible en: https://doi.org/10.10 80/02102412.2016.1140393 (accedido: 27-5-2020).

Aranzadi, Dionisio. (1976). Cooperativismo Industrial Como Sistema, Empresa y Experiencia. Tesis Doctoral. Universidad de Deusto. Bilbao.

Arderiu, Enrique (1980). «El balance social: integración de objetivos sociales en la empresa». Publicaciones de la Real Academia de Ciencias Económicas y Financieras. Disponible en: https://racef.es/archivos/discursos/098_web.pdf (accedido: 27-5-2020).

Ax, Christian y Marton, Jan (2008). «Human capital disclosures and management practices». Journal of Intellectual Capital, 9, 3, 433.

Beattie, Vivien; Mcinnes, Bill y Fearnley, Stella (2004). «A methodology for analysing and evaluating narratives in annual reports: a comprehensive descriptive profile and metrics for disclosure quality attributes», Accounting Forum, 28, 205-236.

Beretta, Sergio; Bozzolan, Saverio y Trombetta, Marco (2007). «Forward looking disclosures and analysts' forecasts: a study of cross-listed european firms». Comunicación presentada al 30 Congreso de la EAA, Lisboa.

CAmpBell, D. (2004). «A longitudinal and cross-sectional analiysis of environmental disclosure in UK companies: a research note». The British Accounting Review, 36, 107-117.

Castro García, Carmen; Álvarez Tardio, Beatriz (s.f.). «La igualdad en la responsabilidad social de las empresas». Disponible en: www.singenerodedudas.com, http://www. castello.es/web20/archivos/contenidos/61/D021_Igualdad_RSE.pdf (accedido: 27-52020).

CEA, José Luis (1980). «La teoría del balance social». Cuadernos universitarios de planificación empresarial, 6, 355-379.

Clarkson, Peter M.; Li, Yue; Richardson, Gordon D. y Vasvari, Florin P. (2008). «Revisiting the relation between environmental performance and environmental disclosure: An empirical analysis». Accounting, Organizations and Society, 33, 4 y 5, MayJuly, 303-327. 
Compromiso Empresarial (2018). "Top Employers 2018: Las mejores 94 compañías para trabajar en Espańa.» CE (Revista Compromiso empresarial). Disponible en: https:/www.compromisoempresarial.com/rsc/2018/02/dime-donde-vives-y-te-direcomo-concilias/ (accedido: 27-5-2020).

De la Cuesta González, Marta; Valor Martínez, Carmen (2003). «Responsabilidad social de la empresa: Concepto, medición y desarrollo en España" Boletín económico de ICE, Información Comercial Español, págs. 7-20, Disponible en: http://www. revistasice.info/cachepdf/BICE_2755_0719_843B2AFA16833BD45F65BF48332 D2587.pdf (acceso 11/11/2019).

Departamento de Empleo y Politicas Sociales del Gobierno Vasco (s.f.). Disponible en: https:/www.euskadi.eus/que-es-la-conciliacion-personal-familiar-y-laboral/ web01-a2concil/es/ (accedido: 27-5-2020).

Diaz Garcia, María Cristina (2007). Influencia del género en los recursos y resultados de las pequeñas empresas. Ed. Consejo económico y social, colección estudios núm. 203, Madrid.

EOI (Escuela de Organización Industrial) (2006). La responsabilidad social corporativa: las politicas familiarmente responsables de las empresas en España. Escuela de negocios Disponible en: https://www.eoi.es/es/savia/publicaciones/19556/laresponsabilidad-social-corporativa-las-politicas-familiarmente-responsables-de-lasempresas-en-espana (accedido: 27-5-2020).

Fabregat Monfort, Gemma (2009). Los planes de igualdad como obligación empresarial. Ed. Bomarzo, Albacete. Pág. 7-33.

Fernández García, Ricardo (2010). Responsabilidad Social Corporativa. Ed. Club Universitario.

Friedan, Betty (1983). La segunda fase. Editorial Plaza \& Janes editores, S.A.

Gamerschlag, Ramin; Möller, Klaus y Verbeeten, Frank (2011). "Determinants of voluntary CSR disclosure: empirical evidence from Germany». Review Management Sciences. Disponible en: https://link.springer.com/article/10.1007/s11846-0100052-3 (accedido: 27-5-2020).

Garcia Sanchez, Isabel María; Prado Lorenzo, José Manuel; Frias Aceituno, José Valeriano (2013) «Información social corporativa y sistema legal». Revista europea de Dirección y Economía de la empresa 22,4, 186-202, Disponible en: https://www.elsevier. es/es-revista-revista-europea-direccion-economia-empresa-346-articulo-informacionsocial-corporativa-sistema-legal-S1019683813000358 (accedido: 27-5-2020).

Garcia, Héctor (2018). «Deporte y trabajo, ¿conciliación en las empresas?» CE (Revista Compromiso empresarial). Disponible en: https://www.compromisoempresarial. $\mathrm{com} / \mathrm{rsc} / 2018 / 03 /$ deporte-y-trabajo-conciliacion-en-las-empresas/ (accedido: 27-52020).

Garcia, Sergio L. (2017). "Las 8 medidas que te ayudarán a conciliar hijos y trabajo en 2017»CE (Revista Compromiso empresarial). Disponible en: https://www. compromisoempresarial.com/rsc/2017/02/las-8-medidas-que-te-ayudaran-a-conciliarhijos-y-trabajo-en-2017/ (accedido: 27-5-2020).

García Sánchez, Isabel María, Martín Zamora, María Pilar y Rodríguez Ariza, Lázaro (2020). «La obligación de ser socialmente transparente: El estado de información no financiera». Revista de Contabilidad y Tributación. CEF, 443, febrero. Madrid.

Garijo, Marta (2018). «Excedencias para cuidar a familiares: el 90\% las piden mujeres». Business insider España. Disponible en: https://www.businessinsider.es/excedenciascuidar-familiares-90-piden-mujeres-187682 (accedido: 27-5-2020). 
Giner Inchausti, Begoña (1995). La divulgación de información financiera: una investigación empírica. ICAC, Ministerio de Economía y Hacienda. Madrid.

Gobierno de España (2017). Plan de acción nacional de empresas y derechos humanos http://www.exteriores.gob.es/Portal/es/PoliticaExteriorCooperacion/Derechos Humanos/Documents/170714\%20PAN\%20Empresas\%20y\%20Derechos\%20 Humanos.pdf (accedido: 27-5-2020).

Gómez Urrutia, Verónica y Jiménez Figueroa, Andrés (2015). «Corresponsabilidad familiar y el equilibrio trabajo-familia: medios para mejorar la equidad de género", Polis Revista Latinoamericana. Disponible en: https://journals.openedition.org/polis/10784 (accedido: 27-5-2020).

Gray, R.; Kouhy, R. y Lavers, S. (1995). "Methodological themes. Constructing a research database of social and environmental reporting by UK companies». Accounting, Auditing and Accountability Journal, 8, 2, 78-101.

GRI (Global Reporting InITIATIVe) (2018). Integrating the SDGs into Corporate Reporting: A Practical Guide.

GRI (Global Reporting Initiative) (2020). Using corporate reporting to strengthen sustainable development goals.

Grijelmo, Alex (2018) «No es Sexista la lengua, sino su uso». El país. Disponible en: https://elpais.com/cultura/2018/02/23/actualidad/1519389008_808351.html (accedido: 27-5-2020).

Guillamón, Encarna (2011). «Corporate governance, future news and forward-looking information». 34 congreso EAA (European Accounting Association), Roma, Italia.

Holsti, O.R. (1969). Content analysis for the Social Sciences and Humanities. Wesley Publishing Company, Readind, MA.

IBERCAMpus (2017). "Casi 10.000 empresas sacan informes de RSC con sello GRI». Ibercampus.es. Disponible en: https://www.ibercampus.es/casi-10000-empresas-sacaninformes-de-rsc-con-sello-gri-34072.htm (accedido: 27-5-2020).

iFAC (International Federation of Accountants) (2020). Building a Coherent, Global Approach to Corporate Reporting. April 2. Disponible en: https:/www.ifac.org/ news-events/2020-04/building-coherent-global-approach-corporate-reporting (accedido: 27-5-2020).

IzQUierdo, Robín (2017). «Ventajas y formas de conseguir la conciliación laboral en tu empresa» Ehorus, Disponible en: https://ehorus.com/es/conciliacion-laboral/ (accedido: 27-5-2020).

Larrinaga González, Carlos; Luque Vílchez, Mercedes (2017). «Actualidad sobre la Directiva 2014/94/UE de información no financiera en España. Revista contable, Sección Estado financieros, 56, Ed. Wolters Kluwer.

Leiva Gomez, Sandra y Comelin Fornes, Andrea (2015). "Conciliación entre la vida familiar y laboral: Evaluación del programa IGUALA en una empresa minera en la región de Tarapacá». Polis Revista Latinoamericana, Ed. Centro de investigación Sociedad y Políticas Pública (CISPO), Disponible en: http://dx.doi.org/10.4067/ S0718-65682015000300016 (accedido: 27-5-2020).

LEY I I/20I8, de 28 de diciembre, por la que se modifica el Código de Comercio, el texto refundido de la Ley de Sociedades de Capital aprobado por el Real Decreto Legislativo 1/2010, de 2 de julio, y la Ley 22/2015, de 20 de julio, de Auditoría de Cuentas, en materia de información no financiera y diversidad. Disponible en: https://www. boe.es/buscar/doc.php?id=BOE-A-2018-17989 (accedido: 27-5-2020). 
LEY 39/1999, de 5 de noviembre, para promover la conciliación de la vida familiar y laboral de las personas trabajadoras. Disponible en: https://boe.es/buscar/pdf/1999/ine-A1999-21568-consolidado.pdf (accedido: 27-5-2020).

Ley Orgánica 3/2007, de 22 de marzo, para la igualdad efectiva de mujeres y hombres. Disponible en: http://www.boe.es/boe/dias/2007/03/23/pdfs/A12611-12645.pdf (accedido: 27-5-2020).

Martín Artiles, Antonio; Carrasquer Oto, Pilar (2005). «La política de conciliación de la vida laboral y familiar en la negociación colectiva. Un aspecto de la estrategia europea de empleo». Cuadernos de Relaciones laborales. 23, 1, 131-150, ISSN 11318635. Disponible en: https://ddd.uab.cat/record/34871 (accedido: 27-5-2020).

Menéndez Llaneza, Carmen (s.f.) «Conciliación de la vida familiar y laboral: presentación y comentarios de la Ley 39/1999, de 5 de noviembre». Federación de industrias textil-piel, quimicas y afines de CC.OO. Madrid. Disponible en: http://usuaris.tinet. cat/jmcf/Legislacio/Llei\%20de\%20conciliacio\%20familiar\%20doclibro.pdf (accedido: 27-5-2020).

MESS (Ministerio de Empleo y Seguridad Social) (2014). Estrategia española de responsabilidad social de las empresas 2014/2020. Disponible en: http://www.mitramiss. gob.es/es/rse/eerse/index.htm (accedido: 27-5-2020).

MSCbS (Ministerio de Sanidad, Consumo y Bienestar Social) (2018). Guia de ayudas sociales y apoyo a las familias. Disponible en: https://www.mscbs.gob.es > ssi , familiasInfancia > pdf , guiadeayudaspar... (accedido: 27-5-2020).

Miller, Katharine (2016). "Cumplir o explicar las políticas de diversidad, la (nueva) exigencia del informe de gestión». CE (Revista Compromiso empresarial). Disponible en: https://www.compromisoempresarial.com/rsc/2016/04/cumplir-o-explicar-las-politicasde-diversidad-la-nueva-exigencia-del-informe-de-gestion/ (accedido: 27-5-2020).

Milne, Markus J. y Adler, Ralph W. (1999). "Exploring the realiability of social and environmental disclosures content analysis». Accounting, Auditing and Accountability Journal, 12, 237-256.

Molina Gonzalez-Pumariega, Rocío (2004). La protección juridica de la mujer trabajadora. Madrid: Concejo económico y social, Colección estudios núm. 162, 107-123.

Musich, Shirley; Hook, Dan; BaAner, Stephanie y Edington, Dee W. (2006). «The association of two productivity measures with health risks and medical conditions in an Australian employee population». American Journal of Health Promotion, 20, 353363. Disponible en: https://www.ncbi.nlm.nih.gov/pubmed/16706007 (accedido: 27-5-2020).

Neuendorf, Kimberley A. (2002). The content analysis guidebook. Sage publications, London.

OIT (Organización Internacional del Trabajo) (2006). Declaración Tripartita de Principios sobre las Empresas Multinacionales y la Politica Social. Disponible en: http:// www.ilo.org/public/libdoc/ilo/2006/106B09_303_span.pdf (accedido: 27-5-2020).

Orellana Cano, Ana María (2002). «Medidas para promover la conciliación de la vida familiar y laboral de las personas trabajadoras. Análisis de las Directivas Comunitarias 92/85 y 96/34 y lagunas en su transposición al ordenamiento interno español». Revista del Ministerio de Trabajo e Inmigración, 37.

ORSC (Observatorio de Responsabilidad Social Corporativa) (2016). «Divulgación de información no financiera, Transposición Directiva Europea 2014/95». Disponible en: https://observatoriorsc.org/wp-content/uploads/2016/11/Informacion no_financiera_propuestaOBRSC_nov2016.pdf (accedido: 27-5-2020). 
Owusu-Ansah, Stephen y Yeoh, Joanna (2005). «The effect of Legislation on Corporate Disclosure Practices». Abacus, vol. 41, n. ${ }^{\circ} 1$.

Perdiguero, G. Tomás (2003). La responsabilidad social de las empresas en un mundo global. Barcelona: Anagrama.

Programa Ó ptima (s.f.). Disponible en: http://habitat.aq.upm.es/bpes/che06/bpes02. html (accedido: 27-5-2020).

Real Decreto Legislativo 2/20 i 5, de 23 de octubre, por el que se aprueba el texto refundido de la Ley del Estatuto de los Trabajadores. Disponible en: https:/www.boe. es/buscar/act.php?id=BOE-A-2015-11430 (accedido: 27-5-2020).

Real Decreto Legislativo 8/20 i 5, de 30 de octubre, por el que se aprueba el texto refundido de la Ley General de la Seguridad Social. Disponible en: https://www.boe.es/ buscar/act.php?id=BOE-A-2015-11724 (accedido: 27-5-2020).

Real Decreto-Ley 3/20i 2, de 10 de febrero, de medidas urgentes para la reforma del mercado laboral. Disponible en: http://www.boe.es/boe/dias/2012/02/11/pdfs/BOEA-2012-2076.pdf (accedido: 27-5-2020).

Rodríguez Álvarez, Sabela (2018). «Lejos de la corresponsabilidad: más del 90\% de las excedencias para el cuidado fueron solicitadas por mujeres». Infolibre Disponible en: https:/www.infolibre.es/noticias/politica/2018/02/04/lejos_corresponsabilidad_las_ excedencias_para_cuidado_fueron_solicitadas_por_mujeres_74744_1012.html (accedido: 27-5-2020).

Rodríguez Escanciano, Susana (2008). La familia en el ámbito jurídico-laboral: situación y protección. Valencia: Tirant lo Blanch.

Rodríguez, Julema; Abreu, José Luis (2009). «Legislación de la responsabilidad social empresarial». Daena (International Journal of Good Conscience); Vol. 4, Pag. 188-228. Disponible en: http://www.spentamexico.org/v4-n2/4(2)\%20188-228.pdf (accedido: 27-5-2020).

RRHHDigital (2019). Las compañías impulsan la natalidad bajo el movimiento «Baby Friendly Companies». RRHHDigital, 12 de noviembre. Disponible en: http://www. rrhhdigital.com/secciones/conciliacion-e-igualdad/139111/Las-companias-impulsanla-natalidad-bajo-el-movimiento-Baby-Friendly-Companies?target=_self (accedido: 27-5-2020).

RuIz, Joaquim (2015). “¿Conoce los distintos estándares sobre RSE?». El blog de prevenContol. Disponible en: http://prevenblog.com/conoces-los-distintos-estandares-sobre-rsel (accedido: 27-5-2020).

Saitua Iribar, Ainhoa; Andicoechea Arondo, Lorea; Albizu Gallastegi, Eneka (2014a). «Human Resources Information in the Management Report of the Top Spanish Companies», Procedia - Social and Behavioral Sciences 109, 171-177, https:// www.sciencedirect.com/science/article/pii/S1877042813050714 (accedido: 27-52020).

Saitua Iribar, Ainhoa; Andicoechea Arondo, Lorea; Albizu Gallastegi, Eneka (2014b). «Divulgación sobre recursos humanos en el Informe de Gestión. Análisis del nivel de comparabilidad y uso de indicadores en las empresas del IBEX35", Boletín de estudios económicos 69 (213), 549-569.

Saitua Iribar, Ainhoa; Albizu Gallastegi, Eneka; Andicoechea Arondo, Lorea (2015). "Human capital information in management reports: An analysis of compliance with the characteristic of the relevance of disclosure», Intangible Capital 11(2), 223-248, https://www.intangiblecapital.org/index.php/ic/article/view/536 (accedido: $27-5-2020)$. 
SaItua Iribar, Ainhoa (2017). «Diversidad de género como parte de la RSC y sostenibilidad en las empresas. Avances para la mejora de la divulgación de información no financiera sobre las políticas de igualdad y sus resultados». Revista Lan Harremanak 38, 178-203, https://www.ehu.eus/ojs/index.php/Lan_Harremanak/article/view/18897 (accedido: 27-5-2020).

S.F. (2018). «La conciliación, segundo factor más importante a la hora de elegir una empresa». ABC Familia. Disponible en: http://www.abc.es/familia/padres-hijos/abciconciliacion-segundo-factor-mas-importante-hora-elegir-empresa-201805230047_ noticia.html (accedido: 27-5-2020).

Soria, Lidia (2018). «Dime dónde vives y te diré cómo concilias». CE (Revista Compromiso empresarial. Disponible en: https://www.compromisoempresarial.com/ rsc/2018/02/dime-donde-vives-y-te-dire-como-concilias/ (accedido: 27-5-2020).

Stratigaki, Maria (2004). The Cooptation of Gender Concepts in EU Policies: The Case of «Reconciliation of Work and Family». Social Politics: International Studies in Gender, State \& Society, Volume 11, Issue 1, Spring Pages 30-56, Disponible en: https:// doi.org/10.1093/sp/jxh025 (accedido: 27-5-2020).

UE (Unión Europea) (2001). Libro Verde. Fomentar un marco europeo para la Responsabilidad social de las empresas Disponible en: https://eur-lex.europa.eu/legal-content/ES/ TXT/?uri=CELEX\%3A52001DC0366 (accedido: 27-5-2020).

UE (Unión Europea) (2014). Directiva 2014/95/UE del Parlamento Europeo y del Consejo, de 22 de octubre de 2014, por la que se modifica la Directiva 2013/34/UE en lo que respecta a la divulgación de información no financiera e información sobre diversidad por parte de determinadas grandes empresas y determinados grupos Texto pertinente a efectos del EEE. Disponible en: https://eur-lex.europa.eu/legal-content/ES/ TXT/?uri=CELEX\%3A32014L0095 (accedido: 27-5-2020). 